\title{
Space to Think: Using Metaphor to Expand Public Thinking about Criminal Justice Reform
}

\author{
Nathaniel Kendall-Taylor ${ }^{1}$ \& Abigail Haydon ${ }^{1}$ \\ ${ }^{1}$ The FrameWorks Institute, Washington DC \\ Correspondence: Nathaniel Kendall-Taylor, The FrameWorks Institute, Washington DC
}

Received: July 29, 2014

Accepted: August 12, 2014

Available online: August 28, 2014

doi:10.11114/smc.v2i2.499

URL: http://dx.doi.org/10.11114/smc.v2i2.499

\begin{abstract}
Metaphor is a vital feature of human cognition that is used both in making and creating meaning. As such, it can play a central role in multiple aspects of communication. In this paper, we synthesize theories of metaphor, culture and framing and argue that metaphors can be designed and tested to serve instrumental functions in communications practice. We discuss and explore this theory through a case study approach — presenting an iterative, multi-method research project that was conducted as part of a larger effort designed to reframe criminal justice reform and expand public discourse on this issue. Through this case study, we synthesize and apply several bodies of theory and offer a methodology for studying metaphors as a communication device. We argue for the power of metaphors as tools in effective communications practice.
\end{abstract}

Keywords: metaphor, crime and criminal justice, public policy, mixed methods design, applied social science

\section{Introduction}

Metaphor is a central feature of cognition (Genter, 1983; Genter, 2008; Kempton, 1987; Hofstadter, 2013). Its centrality in human cognition suggests that, if applied to messaging and translational work, metaphor has the potential to profoundly influence how people understand, interpret and make meaning of information. More specifically, metaphors have the ability to fundamentally restructure the way people reason about social and scientific information and issues (Schön, 1993; Thibodeau \& Boroditsky, 2011). As such, metaphors are a vital tool in efforts to shift and expand the interpretational frameworks that people access and employ in processing information and understanding issues. In so doing, they can increase access to new perspectives and shape public discourse - and, through changed discourse, change social policies and alter structural realities (Schram, 1995).

In this article, we synthesize theories of culture, metaphor and communication and illustrate an application of this theory by presenting a case study of an empirical effort to design metaphors to communicate key perspectives from the field of criminal justice reform to members of the American public.

\subsection{Culture, metaphor and communication}

The concept of metaphor as a translational device - what we will here refer to as an explanatory metaphor - is grounded in the theory of cultural models (Holland \& Quinn, 1987; Shore, 1996) According to D'Andrade, "A cultural model is a cognitive schema that is inter-subjectively shared by a social group" (D'Andrade, 1987, p. 12). In this way, cultural models are tools — or consistently implied relationships and assumptions — that individuals use to function as members of cultural groups and make meaning of their social worlds. Functionally, cultural models allow individuals to make sense of an almost infinite range of incoming information expeditiously enough to interact relatively seamlessly with the people and situations that they encounter (Goffman, 1967). The assumption underlying the concept of cultural models is that members of a cultural group rely on a set of shared understandings derived from common experiences, including shared exposure to mainstream media (Quinn, 2005b).

There are several features of cultural models that are key to their definition and their application to the translational work presented here. First, cultural models are tacit. In other words, members of cultural groups do not refer to or use cultural models explicitly or consciously. Instead, these sets of assumptions and understandings are shared and employed without the awareness of their role in organizing thinking and structuring reasoning processes. Second, they are shared. Membership in a given cultural group is defined and facilitated by possession and use of these shared 
meaning structures such that what is taken for granted is taken for granted by all those sharing the model in a way that allows for fluid and seamless interaction. As D'Andrade explains, "everybody in the group knows ... and everybody knows that everyone else knows ... and everybody knows that everyone knows that everyone knows" (D'Andrade, 1987, p. 113). Third, these models are activated when individuals are engaged in cognitive tasks.

Working from this theory of cultural models, our work in developing explanatory metaphors uses readily understood and familiar concepts - metaphor source domains - to activate cultural models and leverage these existing understandings to structure, or restructure, comprehension of abstract, complex or unfamiliar concepts or ideas — the target domain. Metaphors that are able to key into existing cultural models are able to recast unfamiliar concepts in familiar trappings. Based on this function, we argue that metaphors can unlock new meanings and serve as vital tools for communications and translation work. The intentional cuing of cultural models with metaphor towards instrumental ends is the key theoretical innovation of the perspective and methods we describe here.

At the core, our synthesis of culture, metaphor and communication theories posits that people think through culture as shared mental models, that metaphor is a powerful tool in activating and structuring meaning through its ability to cue and collect cultural models, and that metaphors can be intentionally "designed" to selectively activate cultural models in order to effectively and efficiently communicate complex, abstract or unfamiliar concepts. Our challenge, then, is a) to determine which elements of which existing models we want to evoke and b) how to combine them to create metaphors that shift patterns of understanding and reasoning in directions that are productive in relation to specific translational goals. The result of this process is what we call an explanatory metaphor - a research-driven, empirically tested metaphor that captures and distills a concept by using an explanatory framework that fits in with the public's existing patterns of assumptions and understandings. By pulling out salient features of the problem and mapping onto them the features of concrete, immediate, everyday objects, events or processes, the explanatory metaphor helps people organize information into a clear picture in their minds - with the potential to make them better able to reason productively and think critically about policy and media information.

The research approach described here is also grounded in of social movements and policymaking literatures. The confluence of these literatures suggests the need to focus on the ways that community influentials understand a particular issue and how they could be helped to deepen and express their appreciation for that issue. Public policy scholars from Polsby and Wildavsky (1988) to Iyengar (1991) underscore the importance of public opinion in providing politicians with the "safe space" to initiate policy and forge bipartisan consensus. Getting new ways of talking and thinking about an issue into the public discourse is therefore pivotal in moving a legislative agenda. Metaphors can be powerful in this work as they represent "sticky" ways of lifting the salience of specific issues and solutions in the public discourse.

\section{A Case Study: Using Cultural Models and Metaphor to Reframe Criminal Justice Reform}

In the following section, we describe the application of the theory of culture and metaphor described above to the topic of criminal justice reform. We first explored the cultural models that are at play as people think about crime and the criminal justice system. Following this mapping exercise, we designed and tested a set of metaphors that could be used to encourage productive thinking about the way in which the system shapes outcomes and of the potential of a range of reforms to improve the system and public safety. The iterative, mixed-methods approach described below is designed to manage the limitations of any one method. However, we acknowledge that, across these methods, there remain limitations - chief among them the lack of long-term ethnographic research which would allow for more opportunities to observe language use in more natural settings.

\subsection{Cultural Models of Public Safety and the Criminal Justice System}

We conducted a set of 20 in-depth cultural models interviews (Quinn, 2005a) with members of the American public in San Diego, CA, Stockton, CA, and Virginia Beach, VA, in December 2010 and January 2011. Informants were recruited by a professional marketing firm through a screening process designed to capture variation along the domains of ethnicity, gender, age, educational background and political ideology (as self-reported during the screening process). Those working in fields related to criminal justice and public safety were excluded to facilitate our ability to gather data about how the general public, as non-experts, reasons about target concepts. In total, 11 women and nine men were recruited. Ten participants identified as White, five as African American, three as Hispanic or Latino and two as Asian. Six participants self-identified as "conservative," four as "liberal" and the remaining 10 as "middle-of-the-road." The mean age of the sample was 37 years old, with an age range from 20 to 72 .

Informants participated in one-on-one, semi-structured interviews. Consistent with interview methods employed in psychological anthropology (Quinn, 2005a), cultural models interviews are designed to elicit ways of thinking and talking about issues - in this case, ideas about public safety, criminal justice and connections between these ideas. 
Elements of social discourse analysis, cultural models analysis and grounded theory were applied to identify shared cultural models (Glaser \& Strauss, 1967; Quinn, 1987; Strauss \& Corbin, 1990; Strauss, 2012). First, patterns of discourse, or common, standardized ways of talking, were identified across the sample using a grounded theory approach to thematic analysis. These discourses were then analyzed to reveal tacit organizational assumptions, relationships, propositions and connections that were commonly made but taken for granted throughout an individual's transcript and across the sample. In short, our analysis looked at patterns both in what was said (how things were related, explained and understood) as well as what was not said (shared, but taken-for-granted, assumptions).

In addition to these interviews, we conducted a series of six peer discourse sessions with U.S. citizens in March and April 2011 in three cities: Tampa, FL, Baltimore, MD, and Los Angeles, CA. We again recruited participants through a professional marketing firm using the same sampling approach as described above. Each of the six sessions included nine participants.

Peer discourse sessions are a qualitative approach to exploring common patterns of talk that people use in social settings, and how people negotiate and move among these patterned ways of talking in different conversational and social contexts (Manuel \& Kendall-Taylor, 2009). These sessions were designed to examine whether individuals in social group settings employ the same cultural models that were documented in one-on-one cultural models interviews.

Analysis of data from both the one-on-one interviews and the group sessions suggested that Americans hold the following dominant cultural models of crime, public safety and the criminal justice system:

Top-of-mind associations: Informants largely understood public safety in terms of the front-line responders they saw in their everyday lives, including police, fire and other security personnel. In this way, the "system" was modeled synechdotally through its most visible agents.

Government, communities and citizens are responsible: Public safety was understood through a dispersed model of responsibility in which the government, local communities and individuals are responsible for maintaining public safety.

Ideal vs. real: When asked to describe what each branch of the criminal justice system does, informants emphasized the disconnect between what the criminal justice system should be doing and how it actually operates.

Conflicting ideas about the causes of crime: Informants understood the causes of crime through the application of three distinct, conflicting models. The first model attributes crime to individuals who fit one of a set of criminal "personality types." The second explains it as a result of ecological determinants, and the third model understands crime in relation to early experiences and developmental factors.

Rational-actor considerations: Informants' judgment of those who commit crimes hinged upon whether they saw the person as being capable of making a conscious, "rational" decision. If the accused was viewed as a rational actor (which included everyone except very young children and individuals suffering from extreme forms of mental illness), informants saw prison and harsh punishment as the appropriate response. By contrast, if the accused was understood as incapable of logical thought, informants were more likely to consider alternatives to prison.

Opposing logics of "fair" sentencing: Informants believed that "fairness" should determine sentencing, but applied this value in two opposing ways. The uniform model of fairness posited that punishments for crimes should be fixed regardless of the circumstances surrounding the crime. The contrasting contextual model of fairness was premised in the idea that sentencing should consider the unique circumstances of the crime, including the defendant's upbringing, criminal history, mental health, intent and evidence of remorse.

Cultural models and solutions: Our research strongly suggested that the way in which informants understood causation of crime shaped their understanding of effective responses and solutions. When informants employed rational-actor models, they tended to arrive at a very specific and narrow set of solutions that included making punishments harsher and sentences more uniform. On the other hand, when they applied more ecological models to thinking about public safety and criminal justice, they arrived at contextual, policy and resource-based solutions to problems in these domains. This link between causal modeling and solutions recognition and support is the key to the metaphor research described below. Underlying all solutions thinking, however, was a strong assumption that attempts to improve large public systems are largely futile.

\subsection{Two Metaphors for the Criminal Justice System and Criminal Justice Reform}

Based on the cultural models findings described in section 2.1, we established the following key conceptual tasks for explanatory metaphors to accomplish.

1. The metaphor should make the system-ness of the criminal justice system more apparent and concrete. 
2. The metaphor should provide a basis for understanding how contexts (both environmental and institutional ones) shape individuals' actions and choices.

3. The metaphor should help people understand the specific ways that the criminal justice system is inefficient as well as inequitable.

4. The metaphor should make people more articulate about how the criminal justice system in America could be improved.

5. The metaphor should allow people to see solutions at the level of addressing the system rather than through affecting each individual's cost/benefit calculation.

We identified, refined and empirically tested a total of 35 potential explanatory metaphors. Two of these metaphors, Justice Gears and The Justice Maze, emerged as effective in providing a more systemic perspective and supporting productive discussion of the challenges facing the criminal justice system and the reforms necessary to improve this system. The following are presentations of these two metaphors:

Justice Gears: Right now our justice system is stuck using only one gear - the prison gear. Think about how a bicycle works and how it needs to use different gears to work effectively and efficiently -it needs different gears to use in different situations. Right now, the criminal justice system is dealing with a wide variety of situations using only one gear. We need to have other gears for people who come into the system, like mental health services, addiction services, or juvenile justice services. We need to change the criminal justice system to make sure it has different gears for different purposes and that it's set up in a way that it uses the right gear in the right situation. If we do this we can improve outcomes and all get to where we need to go.

The Justice Maze: Even in the most difficult mazes, there's a way to get in and out. But the criminal justice system that we have now is designed with too many gaping entry points and without enough paths that come out. A lot of people, no matter where they come into the criminal justice system, get stuck in a path that goes straight to prison and doesn't have a way out. We know that we need to make other routes available. We need paths to mental health services, addiction services, or juvenile justice services and we need these to be two-way paths so that people can get to where they need to go. We need to take the justice maze and redesign so that it clearly lays out multiple routes to get people where they need to go in the most effective and efficient way possible.

Below, we briefly discuss the methods by which we identified, developed and empirically tested the Justice Gear and Justice Maze explanatory metaphors and the findings from each stage of this process. We conclude with a set of observations and next steps for those thinking about the role of metaphor in strategic communications.

\subsection{Evidence from On-the-Street Interviews}

We conducted 42 on-the-street interviews with people in Atlanta, GA, and Baltimore, MD. Informants in these interviews were selected to represent variation in demographic categories including age, gender, race/ethnicity and political ideology. These interviews tested the ability of seven candidate metaphors to enable more productive and robust discussions of the criminal justice system.

Informants were first asked a set of questions about how the criminal justice system works; what, if anything, might be wrong with it; and how it might be improved. They were then presented with one of the candidate explanatory metaphors. After the metaphor was presented, they were asked the earlier questions in a rephrased form. Two researchers independently analyzed the resulting video data, looking for patterned ways in which each of the candidate metaphors affected thinking and talking about the criminal justice system. The analysis also focused on isolating the reasons why each of the tested metaphors was having its respective effects.

Justice Gears and The Justice Maze were inspired by the promising performance in on-the-street interviews of other metaphors. Justice Gears grew out of the metaphor Justice Triage, which sought to explain systemic inefficiencies by comparing the justice system to an ineffective hospital; it was designed to take advantage of the positive results observed in the Triage metaphor while avoiding some of that metaphor's less productive effects (for example, the entailment that criminals are "sick"). Justice Maze was inspired by the positive effects of Justice Highway, which prompted participants to talk about how the system is designed and built and to connect structural conditions to individual outcomes.

\subsection{Evidence from the Quantitative Experiment}

Using the results from the on-the-street interviews to winnow the set of candidate metaphors and refine existing iterations, we designed a large-scale experimental survey to quantitatively assess the efficacy of the refined set of metaphor candidates. This test, a head-to-head comparison using random assignment techniques and a nationally representative sample of 1,200 Americans, allowed us to chart how well each explanatory metaphor functioned to 
achieve the goals described above. The sampling strategy and assignment techniques were used to assure generalizability and to determine causal links between independent and dependent variables. Five metaphors and one null control condition were tested. Respondents randomly assigned to the null control group were shown no metaphor but answered all outcome questions. The outcome measures consisted of 27 multiple-choice questions that tested the metaphors' performance in relation to two areas: (1) How did people understand the problems facing the criminal justice system, and (2) could they apply the metaphor to thinking about several issue areas, including prison overcrowding, unequal treatment, juvenile justice, and overall efficiency. Figure 1 provides the results from the experiment.

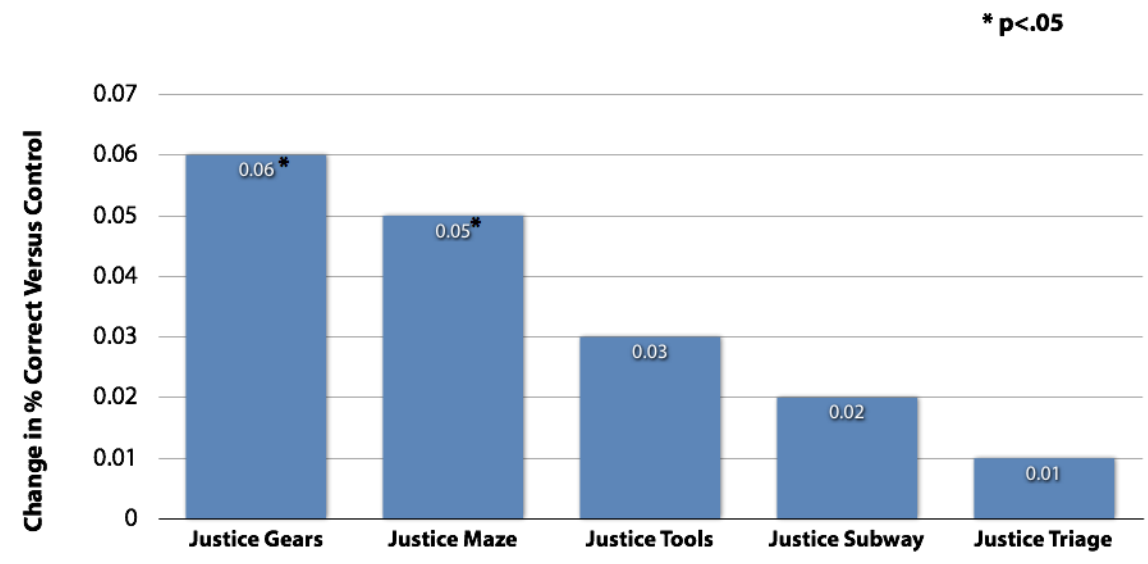

\section{Metaphor \\ Figure 1. Results of Metaphor Experiment}

The metaphor effectiveness scores of both Justice Gears and The Justice Maze were statistically significant at the $p$ $<.05$ level in relation to the control and the lowest two performing metaphor. Based on these results and those from the earlier on-the-street interviews, we took these two metaphors forward into the final stage of research: Persistence Trials.

\subsection{Evidence from Persistence Trials}

In a Persistence Trial, an initial pair of participants is presented the explanatory metaphor, first as text and then conversationally by the researcher. The participants then discuss the explanatory metaphor with the moderator before teaching it to a subsequent pair of participants. Following the transfer, the second pair explains the explanatory metaphor to a third pair. Finally, the first pair returns to hear the transmitted metaphor from the third pair. This last step allows us to see whether the metaphor has persisted over the session and to enlist participants in explaining any changes that may have occurred to the metaphor. We conducted Persistence Trials in Philadelphia, Pennsylvania and Jacksonville, Florida, for a total of six sessions with 36 participants. In each city, there was one session devoted solely to African-American participants. All other sessions were recruited to represent variation in race, among other demographic variables.

\subsubsection{Application of the Justice Gears Metaphor}

Participants in Persistence Trials applied the Justice Gears metaphor in the following ways.

A focus on systems. At a general level, the metaphor was highly effective in channeling people's focus and attention towards systems and structures and away from individual-level thinking about the causes of and solutions to crime. The mechanical nature of the gears metaphor appeared instrumental in this channeling. Thinking about a machine and mapping this mechanistic thinking onto the issue of criminal justice immediately appeared to focus people's attention on the functions and fit of different parts of the system.

The need for alternative approaches. One of the most striking features of the metaphor was its effect in helping participants see 1) the need for interventions other than incarceration when dealing with offenders, and 2) that prison is not the only way, nor the best way, to improve public safety.

Participant: We have ten gears that we could use, but we're only using one of them! If we broaden what is available and use these other gears, we can gear up and gear down, and move forward and be a little more exploratory in our methods rather than just "put them in jail." Prison to me is "one gear."

Participants talked about how gears make a bike work more efficiently and effectively over different types of terrains. 
This notion of "terrain" became associated with the factors associated with criminal offenses that should be taken into account by the adjudication process. The following interaction shows how the bicycle domain structured conversation in this way:

Participant 1: There are many things we think are wrong with the criminal justice system. If we want different outcomes, we need to have different sorts of approaches.

Participant 2: How would a different gear lead to a different outcome?

Participant 1: Because if you're in first gear, that's an easier gear to pedal on, whereas if you're on 15th gear, it's harder to pedal on that — but it's appropriate depending on the terrain. If you're going uphill you don't want it on gear 15, because it's going to be harder and it's not going to work.

Participant 2: I'm trying to relate this to breaking out of the system. Maybe the accused person doesn't need to go to a criminal court but needs to get treatment for something.

It is also important to note that, when participants talked about "bicycling on a terrain," it generated discussions of "avenues" and "paths," including "different paths." These discussions of "different paths" frequently let to a focus on the need for alternatives to prison in order to improve efficiency and, ultimately, outcomes.

Participant: Bikes have different gears and we use different gears for different reasons. When you're going uphill you don't want the highest gear on the bike, you want the lowest gear, so each gear has its purpose. Just like with the bike, we think there could be different gears in the criminal justice system, in order to send people down the right path, in other words, depending on what the terrain is.

Outcomes are key. As mentioned above, Justice Gears led participants to discuss paths, travel and frequently, destinations. When applied to the criminal justice system, talk about destinations focused participants' attention on the system's outcomes - and on the fact that effective reform should focus not only on punishment but also on improving outcomes for individuals and society.

Participant: Almost everything you experience going through that one gear is punitive. Some people are stronger than others. The experience doesn't affect them as much while other people are totally broken and they're left that way. I don't know how you got there, but that doesn't make you better.

Participant: People are being channeled down this one pike, this one path, but maybe there could be different outcomes if you evaluated the reasons for the crime rather than evaluating just the crime.

Improving the fit between the offense and response would improve effectiveness. The concept of gears proved powerful in helping participants generate lists of problems and solutions and in focusing on the importance of having appropriate "fit" between these concepts. Participants drew on the common understanding that gears must fit together tightly in order to work and mapped this onto areas where the criminal justice system needed improvement. This discussion tended to highlight methods for changing or repairing the system, rather than demolishing or destroying it and starting over from scratch. Two of the gears that needed to "fit" in the criminal justice system were offenses and responses to those offenses.

Participant: We thought about the criminal justice system and started to come up with some problems that we saw — things like inequities and prisoners getting treated poorly. If you were to sit and talk about it for a little while, you'd come up with a bunch of ideas of what is wrong with the system, but [what] we're talking about is a gear approach to correcting some of these problems to make the criminal justice system fairer, more efficient, less expensive, better at serving the public and better at serving prisoners. So we're using this gear approach to think about what some of the problems are but also in thinking of how we can fix some of those problems. We need to mesh those [problems and solutions] together, in the way that gears in a car transmission mesh together.

Productive critiques of the system. Our earlier research on cultural models suggested that Americans have an easy time complaining about the actions of "crooked cops" or "old racist judges." However, without the aid of a framing tool, people have a harder time lodging critiques at the systems level. A powerful effect of Justice Gears was that it structured criminal justice criticisms at the systems level, in ways that aligned with the critiques offered by criminal justice experts and advocates.

Participant: Some of the things you hear regularly about the system, is things like mental health and addiction and background and race and how all these factors come into play, and people being sent to specific places for incarceration because certain judges are getting paid. That all these things are part and parcel of gears [of the system] that aren't meshing right. What can we do to get some of the gears to mesh better, or figure out what 
gear that one is supposed to attach to?

Heightened visibility of bias - both economic and racial. In conversations following exposure to the metaphor, participants were articulate and willing to talk about economic biases in the criminal justice system, mainly in the area of sentencing and adjudication. The metaphor also helped participants discuss racial biases inherent in the current system. However, while these discussions of racial bias shifted in relation to unprimed conversations observed in previous research, they remained less frequent than those of class.

Participant: For white people, they're using all the gears. For African Americans, they're using one gear.

--

Participant: I know for me some of the biggest problems that I see and hear about is the fact that there seems to be, at least from the punishment phase, there are two different types of justice in terms of who you are, what you look like, whether or not you've had the money to pay a good attorney who will fight to the death to lessen the charges. It seems to me the punishment phase [is] very unfair.

--

Participant: I thought the terrain was representing the loopholes, the political hoops that you jump through going through the criminal justice system. Because that terrain is going to look different for different people. It is what it is. It's going to look different for a black kid from the city than it is for Joe from Swarthmore.

\subsubsection{Inoculation by the Justice Gears Metaphor}

Justice Gears also showed an ability to inoculate against — or channel people's thinking away from — several powerful default cultural models that move people in unproductive directions when thinking and talking about the criminal justice system.

Against individualist thinking. When people focus on the actions and motivations of individuals as the causes of events, it becomes difficult to recognize the appropriateness and importance of systems-level reforms. Justice Gears appeared to inoculate against such an individualistic perspective and channel attention towards systems.

Participant: What we're trying to convey is that what's wrong with the criminal justice system is the system there are many things that are wrong with the criminal justice system and they all lead to the same outcome. So if we want to have different outcomes, we need to have different sorts of approaches, different paths.

Against deterministic thinking. Justice Gears also invigorated the sense that - just as there are ways to fix gears, bikes and engines - there are concrete steps we can take to improve the criminal justice system. In so doing, it inoculated against the tendency to view the system as too big, complicated and opaque to meaningfully improve.

Participant 1: What can we do to improve the criminal justice system? It's like a giant machine that has broken down. And all these parts of the criminal justice system need to be fixed. If you don't fix this [one] part, this [other] part will never get fixed.

Participant 2: Because the engine, the machine won't run - the parts aren't working correctly.

Participant 1: So you got your arresting officers, [they] are the first part, then the court and the judge are the next, then the punishment for the crime. Then the prison and the prison guards, and all of that, so each division needs to be fixed. Each one has to be fixed in order to have the criminal justice system work.

Against rational actor thinking. In unframed discussions, we found that people often employ a rational actor model of crime, in which individuals commit crimes based on a rational and intentional cost/benefit analysis. According to this assumption, people commit crimes because they have determined that the benefits of such actions outweigh the costs. The following exchange between two African-American participants demonstrates the power of this cultural model.

Participant 1: It's never going to be perfect and it's always going to have to have many faults because of us, because of people, because of society. So listen, "stop doing stupid stuff and getting in trouble and going to jail," and guess what? The criminal justice system changes, and becomes more manageable. And at the rate we have — and especially black men — the rate that we get in trouble and go to jail....

Participant 2: Why is that?

Participant 1: To me, that's the result of society and economics, and that leads into that issue — but it's not an excuse.

Participant 2: We all have choices....

Despite the strength of this mode of thinking, the Justice Gears metaphor, with its strong systems focus, 
disrupted the rational actor model by making more visible the broader social ecology that produces crime.

Participant: What we're doing here is comparing the justice system to a ten-speed bicycle that has gears on it. And the issue is that the criminal justice system can be very difficult, and it kind of has a revolving door type of atmosphere to it. People come in and they see the judge and there's a public defender, and they're in and out, when actually there are mitigating circumstances that could lessen the severity of the crime because of other factors that might have contributed to why the crime was done, but that's not being taken into account by the criminal justice system.

\subsubsection{Self-Correction of the Justice Gears Metaphor}

Self-correction refers to an explanatory metaphor's ability to "snap back" to its initial form following a deterioration or mutation of the concept in discussion. An important measure of an explanatory metaphor's strength, self-correction occurs when certain features fall out of conversation and then re-assert themselves in subsequent discourse without being re-cued by the moderator. When communicated in the public sphere, explanatory metaphors are likely to break down. It is therefore important that a concept have sufficient internal coherence to recover from devolutions - to encourage people to arrive at key understandings despite partial or inaccurate communication of the explanatory metaphor.

There were several occasions in which Justice Gears devolved to some degree and then "snapped back" into its original form. In one session, the functionality of the gearing - that it actually matters for the efficient functioning of the system - dropped out as the metaphor was passed between the second and third groups. However, this key feature of the metaphor reasserted itself in the third group's conversation without prompting by the moderator. The following interaction shows how the third group found their way back to this important feature of the metaphor:

Participant 1: On the one hand, we're using the analogy, and I'm thinking, you use gears on a bike because it helps you pedal faster.

Participant 2: Well, it helps you to be more effective. Could we be more effective if we were utilizing more gears on this bike?

Participant 1: Absolutely. I think what you need, you use the analogy along with the types of crimes we're talking about, and we say, okay, some of them all start out in one gear or another depending on what kind of crime it is, but based on the rehabilitation, you're either going uphill or downhill or you're going to a straightaway, and you need to go use the different gears in order to match the crime, the person and the time.

\subsubsection{Communicability of the Justice Gears Metaphor}

Communicability refers to the faithfulness of the transmission of the explanatory metaphor among participants.

The notion of "gears" and a bicycle with only "one gear" persisted easily across the transmissions. One reason for the "stickiness" of the metaphor is the wide range and large number of related idiomatic expressions, such as "stuck in a rut," "changing gears," "different path," "mesh together," "cross purposes," "fixed" and "overhauled." These expressions show the richness of the gears domain - a feature that is essential to a highly communicable metaphor.

Another characteristic of a metaphor with a high degree of communicability is that, when participants use it or talk about it, they make gestures with their hands and fingers (Alibali \& Kita, 2010; Alibali, Spencer, Knox, \& Kita, 2011; Krauss, Chen, \& Shawla, 1996) In the case of Justice Gears, participants frequently illustrated "gears" working, turning, and, fitting together with their hands. When such gestures accompany a metaphor, they indicate that the metaphor has been powerfully incorporated into deep parts of the participant's cognition and meaning-making - in short, that the metaphor has a high degree of "thinkability." This is an important aspect of communicability and of the metaphor's more general power as a communications tool.

\subsubsection{Application of the Justice Maze Metaphor}

Persistence Trials showed that participants applied The Justice Maze metaphor in the following ways.

A contextual picture of crime. The Justice Maze productively channeled and checked the public's dominant focus on individuals and individual rational decisions as the causes of and solutions to issues of public safety. Put simply, by focusing people's attention on the structure that people move through (the maze), the metaphor shifts people's attention from the individual to the contexts and systems in which they are embedded. From this perspective, people can better appreciate arguments about the biases that are built into the current system and the need to address these biases at a systems and structural level. This is the major utility of The Justice Maze and a vital conceptual task in reframing the issue of criminal justice.

Moderator: So they [the previous pair of participants] put drug rehabilitation centers and mental health service and the juvenile justice system in the maze. Are those part of the maze? 
Participant: I think they can also be little roads out of the maze. You can be in the maze and then you can go to the juvenile justice system and someone says, "we're going to get you some legal help because you were 17 when you committed this crime. We're going give you the benefit of the doubt. We're going to expunge your record, here's your do-over card — now go do better."

Participant: The criminal justice system is sometimes looked at as a cycle and a maze because once inside the system, it's hard to get out. There are factors that people bring into the prison like mental illness and drugs, and the job of the prison is to get them out to be productive citizens and break the cycle while they are inside. But the problem is, this system doesn't exist, and the cycle just keeps going. They don't offer job training skills so people end up right back in the system.

Alternatives are needed. As with Justice Gears, The Justice Maze was highly effective at getting people to see how alternatives to incarceration are needed. The language around "only one path" was particularly effective at structuring productive critiques of the fact that prison is currently the "only path" or "the only end" in the system. The idea of "dead ends" was also highly effective in getting people to recognize and robustly discuss recidivism - the fact that once you have been in prison there is no route to a productive life.

Participant: The maze system is the system and prison is the only exit, but it's not really an exit — it's the end result. And everybody is lumped together. You're going to go through the maze, and when you come around that corner, you're going to get dumped out into prison.

The current system is inefficient and ineffective. As with Justice Gears, The Justice Maze gave people cognitive and linguistic tools for characterizing what is wrong with the criminal justice system and what needs to change. In the main, this concerned the adjudication and the incarceration/rehabilitation processes.

Participant: What I got out of the maze is that something needs to be fixed. There are roadblocks everywhere for people going into the system. And then there's no way out of that maze.

Participant: I would think it's, you're re-filtering back into the maze people who are, they know they're going back into the roadblocks and the struggles they went into before.

Participant 2: Habitual offenders, there needs to be something done for them. Sending them back into prison repetitiously - there has to be a better way to do things.

2.5.6 Inoculation by the Justice Maze Metaphor

Against rational actor thinking. As noted above, Americans frequently attempt to understand criminal behavior through a rational lens and attribute a discrete cost/benefit analysis to offenders. As with Justice Gears, The Justice Maze disrupted this perspective, suggesting other determinants of behavior, such as social conditions, environments or developmental stages.

Participant: There is no redemptive function in that system. If you have a felony, forget it, you have no chance. Once you have a record, you're screwed. It's really hard for you even to get a job. If you don't give a person a chance to provide some kind of livelihood for themselves, you are driving them to a life of crime. So that's the maze, once you're in it you can't get out of it.

Against individual responsibility thinking. Along with the rational actor model comes the notion that the criminal justice system responds purely on the basis of crimes committed and therefore that any differences between groups of Americans are due to shortcomings of those individuals or groups. When reasoning from this model, people explain the predominance of young men of color in the system as due to moral failings, not to systematic and structural discrimination. The maze was effective in inoculating against this way of thinking about group differences by, again, focusing attention on the structures that people move through — on the paths available, rather than on the individuals moving through them. Participants used the maze as a way to talk about how the system's permutations and paths change for different populations, explaining that different people go through different mazes. In this way, participants productively modified The Justice Maze into a notion of justice mazes, as a way of discussing the structural bias of the system. In such discussions, participants not only focused on bias in productive ways, but they grounded and rooted these biases in structural aspects of the system.

\subsubsection{Communicability of the Justice Maze Metaphor}

The Justice Maze was easily communicated and highly persistent. Perhaps most indicative of its communicability was the number of expressions related to paths and structures that participants used, such as "multiple outlets," "multiple options," "exits," "dead ends," "hitting a brick wall," "coming around a corner," "not progressing through," "alternative 
exits," "one way routes" and "roadblocks." More broadly, the underlying conceptual metaphor of "life as a path" or "life as a journey" was highly visible in these discussions and underlay the metaphor's high degree of communicability. Similarly, the conceptual metaphor of "institutions as structures" was also highly effective and sticky as the metaphor was passed between participants. Most of the effectiveness of The Justice Maze stems from the way that these two domains blend in productive ways. The metaphor's path component helps people see individuals and groups in the system, while its structural component contextualizes the way that individuals move through the maze, revealing how the structure shapes outcomes and must be addressed to improve them. The maze metaphor was sticky across the transmissions and did not degrade. This strength in communicability meant that researchers had no chance to observe its ability to self-correct.

\subsection{Recommendations for Using Justice Gears and Justice Maze}

Below we offer a summary of research-based recommendations on the use of Justice Gears and Justice Maze.

Use Justice Gears to communicate about:

- The inefficiencies of the criminal justice system, as they relate to policing, adjudication, and rehabilitation/punishment.

- The need to employ alternative measures to improve public safety and create better outcomes for those who come in contact with the criminal justice system.

- The ways in which the system works differently for different groups of people.

Use The Justice Maze to communicate about:

- How structural aspects of the criminal justice system shape individual and group outcomes.

- Systemic inefficiencies and biases.

- The need for reforms to address structural aspects of the system.

- How the structure of society predetermines inequitable outcomes for some populations.

\section{Conclusion}

As is the case with most systems in American public life, the scope, components, and functioning of the criminal justice system are difficult for many Americans to grasp. As a result, they struggle to consider what can be done to improve the system and address its challenges. Our results suggest that the Justice Gears and Justice Maze explanatory metaphors constitute powerful tools for reframing the conversation about public safety and criminal justice reform. Our development and empirical testing of these metaphors is grounded in an interdisciplinary theory of culture, metaphor and communication that centers on the intentional cuing of cultural models to advance specific translational goals. Positioning metaphors within the universe of available cultural models takes advantage of the nested nature of cultural models and how they are used in thinking. By tailoring metaphors to employ elements of existing models, and empirically testing their utility, we make an important step toward assuring their palatability, uptake and incorporation.

This work, and the theory upon which it is based, suggests several directions for research to extend the use of metaphor as communications devices. First, we see a need for research that investigates the best ways to cue metaphor - for example, through visuals, written language, or oral presentation - and the most effective construction of metaphor iterations. To what extent is understanding broadened by a single sentence or descriptive title? Do explanatory metaphors fare best when embedded in broader explanations or narratives? Future research should also explore whether other communications tools (e.g., exemplars, values) cue and recruit cultural models for instrumental ends, as we have demonstrated with metaphors. Finally, we hope that additional research will investigate the most effective means of diffusing and dispersing empirically-tested communications devices like those presented here - so that they can realize their full potential of broadening public understanding of complex sociopolitical issues.

\section{Acknowledgement}

This research was conducted by the Ford Foundation. Since 1999, the FrameWorks Institute has been developing an applied theory and practice of evidence-based communications research that draws heavily from the cognitive sciences. Credit for a great deal of the thinking presented in this article here goes to our colleagues at the Institute. Special thanks to Michael Erard, who contributed to an early draft of this paper.

\section{References}

Alibali, M. W., \& Kita, S. (2010). Gesture highlights perceptually present information for speakers. Gesture, 10, 3-28. http://dx.doi.org/10.1075/gest.10.1.02ali

Alibali, M. W., Spencer, R., Knox, L., \& Kita, S. (2011). Spontaneous gesture influences strategy choices in problem 
solving. Psychological Science, 22(9), 1138-1144. http://dx.doi.org/10.1177/095679761141772

D'Andrade, Roy. (1987). A folk model of the mind. In D. Holland \& N. Quinn (Eds.), Cultural models in language and thought (pp. 112-150). Cambridge: Cambridge University Press.

Genter, D. (1983). Flowing waters and teeming crowds: Mental models of electricity. In D. Genter \& A. Stevens (Eds.), Mental models (pp. 99-129). Hillsdale, NJ: Lawrence Erlbaum Associates

Genter, D., \& Bowdle, B. (2008). Metaphor as structure-mapping. In R. Gibbs (Ed.), Cambridge handbook of metaphor and thought (pp. 109-128). Cambridge, England: Cambridge University Press.

Glaser, B. G., \& Strauss, A. L. (1967). The discovery of grounded theory: Strategies for qualitative research. Chicago, IL: Aldine Publishing Company.

Goffman, E. (1967). Interaction ritual: essays in face-to-face behavior. New Brunswick, New Jresey: Transaction Publishers.

Hofstadter, D., \& Sander, E. (2013). Surfaces and essences: Analogy as the fuel and fire of thinking. New York, NY: Basic Books.

Holland, D., \& Quinn, N. (1987). Cultural models in language and thought. Cambridge, England: Cambridge University Press.

Iyengar, S. (1991). Is Anyone Responsible? How Television Frames Political Issues. Chicago, IL: University of Chicago Press.

Kempton, W. (1987). Two theories of home heat control. In D. Holland \& N. Quinn (Eds.), Cultural models in langauge and thought (pp. 222-242). Cambridge, England: Cambridge University Press.

Krauss, R., Chen, Y., \& Shawla, P. (1996). Nonverbal behavior and nonverbal communication: What do conversational hand gestures tell us? In M. Zanna (Ed.), Advances in experimental social psychology (pp. 389-450). San Diego, CA: Academic Press.

Lakoff, G., \& Johnson, M. (1980). Metaphors we live by. Chicago, IL: The University of Chicago Press.

Manuel, T., \& Kendall-Taylor, N. (2009). From focus groups to peer discourse sessions: The evolution of a method to capture language, meaning, and negotiation. New Directions for Youth Development, 124, 61-69. http://dx.doi.org/10.1002/yd.325

Polsby, N., \& Wildavsky, A. (1988). Presidential elections, 7th Edition, New York, NY: The Free Press.

Quinn, N. (1987). Convergent evidence for a cultural model of American marriage Cultural models in language and thought (pp. 173-192). Cambridge, England: Cambridge University Press.

Quinn, N. (2005a). Finding culture in talk: A collection of methods. New York, NY: Palgrave MacMillan.

Quinn, N. (2005b). How to reconstruct schemas people share, from what they say. In N. Quinn (Ed.), Finding culture in talk: A collection of methods. New York, NY: Palgrave MacMillan.

Schön, D. (1993). Generative metaphor: A perspective on problem-setting in social policy. In A. Ortony (Ed.), Metaphor and though (pp. 137-163). Cambridge, England: Cambridge University Press.

Schram, S. F. (1995). Words of welfare: The poverty of social science and the social science of poverty. Minneapolis, MN: The University of Minnesota Press.

Shore, B. (1996). Culture in mind: Cognition, culture, and the problem of meaning. Oxord, England: Oxford University Press.

Strauss, A. L., \& Corbin, J. M. (1990). Basics of qualitative research: Grounded theory procedures and techniques. Thousand Oaks, CA: Sage Publications.

Strauss, C. (2012). Making sense of public opinion: American discourses about immigration and social programs. Cambridge, UK: Cambridge University Press.

Thibodeau, P., \& Boroditsky, L. (2011). Metaphors we think with: The role of metaphor in reasoning. PLoS ONE, 6(2), e16782. http://dx.doi.org/10.1371/journal.pone.0016782

\section{(cc) BY}

This work is licensed under a Creative Commons Attribution 3.0 License. 